International Journal of Pure and Applied Mathematics

Volume 104 No. 3 2015, 299-311

ISSN: 1311-8080 (printed version); ISSN: 1314-3395 (on-line version)

url: http://www.ijpam.eu

doi: http://dx.doi.org/10.12732/ijpam.v104i3.2

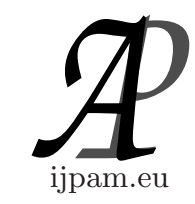

\title{
HYERS-ULAM-RASSIAS STABILITY OF LIE *-DERIVATIONS OF A CUBIC FUNCTIONAL EQUATION WITH THREE VARIABLES
}

\author{
Ick-Soon Chang ${ }^{1}$, Hwan-Yong Shin ${ }^{2} \S$ \\ ${ }^{1,2}$ Department of Mathematics \\ Chungnam National University
}

99 Daehangno, Yuseong-gu, Daejeon 305-764, KOREA

\begin{abstract}
We will prove the general solution of the following cubic functional equation

$$
\begin{aligned}
& 4\{f(2 x+y+z)+f(x+2 y+z)+f(x+y+2 z)\}=27 f(x+y+z) \\
& \quad+f(-x+y+z)+f(x-y+z)+f(x+y-z)+12\{f(x)+f(y)+f(z)\}
\end{aligned}
$$
\end{abstract}

and investigate the stability of a cubic Lie $*$-derivation associated with the given functional equation.

AMS Subject Classification: 39B82, 39B62

Key Words: cubic functional equation, Lie $*$-derivation

\section{Introduction}

In the theory of functional equations, a classical problem is the following: "When is it true that a function which approximately satisfies a functional equation $\varepsilon$ must be close to an exact solution of $\varepsilon$ ?" If the problem accepts a solution, we say that the equation is $\varepsilon$-stable. The first stability problem about group homomorphisms was raised by Ulam [13] in 1940. We are given a group $G$ and a metric group $G^{\prime}$ with netruc $d(\cdot, \cdot)$. Given $\varepsilon>0$, does there exist a

Received: March 17, 2015

(c) 2015 Academic Publications, Ltd.

${ }^{\S}$ Correspondence author url: www.acadpubl.eu 
$\delta>0$ such that if $f: G \rightarrow G^{\prime}$ satisfies $d(f(x y), f(x) f(y))<\delta$ for all $x, y \in G$, then a homomorphism $h: G \rightarrow G^{\prime}$ exists with $d(f(x), h(x))<\varepsilon$ for all $x \in G$ ? Ulam's problem was partially solved by Hyers [5] in 1941. Let $E_{1}$ be a normed space, $E_{2}$ a Banach space and suppose that a mapping $f: E_{1} \rightarrow E_{2}$ satisfies the inequality

$$
\|f(x+y)-f(x)-f(y)\| \leq \varepsilon
$$

for all $x, y \in E_{1}$, where $\varepsilon>0$ is a constant. Then the limit $T(x)=\lim _{n \rightarrow \infty} \frac{f\left(2^{n} x\right)}{2^{n}}$ exists for each $x \in E_{1}$ and $T$ is the unique additive mapping satisfying

$$
\|f(x)-T(x)\| \leq \varepsilon
$$

for all $x \in E_{1}$. Also, if the function $t \longmapsto f(t x)$ from $\mathbb{R}$ to $E_{2}$ for each $x$ is continuous on $\mathbb{R}$, then $T$ is linear. If $f$ is continuous at a single point of $E_{1}$, then $T$ is continuous everywhere in $E_{1}$. Moreover (1) is sharp.

Bourgin [2] was the next author who treated this problem for additive mappings (see also [1]). In [12], Rassias provided a generalization of Hyers theorem which allows the Cauchy difference to be unbounded. Găvruta then generalized the Rassias result in [4] for the unbounded Cauchy difference. Subsequently, various approaches to the problem have been studied by a number of authors.

Also, Jang and Park [6] investigated the stability of $*$-derivations and of quadratic $*$-derivations with Cauchy functional equation and the Jensen functional equation on Banach *-algebra. The stability of $*$-derivations on Banach *-algebra by using fixed point alternative was proved by Park and Bodaghi and also Yang et al.; see [10] and [14], respectively. In particular, the stability of cubic Lie derivations was introduced by Fošner and Fošner; see [3].

Let both $E_{1}$ and $E_{2}$ real vector spaces. Jun and Kim [8] proved that a mapping $f: E_{1} \rightarrow E_{2}$ satisfies the functional equation

$$
f(2 x+y)+f(2 x-y)=2 f(x+y)+2 f(x-y)+12 f(x)
$$

if and only if there exists a mapping $B: E_{1} \times E_{1} \times E_{1} \rightarrow E_{2}$ such that $f(x)=$ $B(x . x . x)$ for all $x \in E_{1}$, where $B$, defined by

$$
\begin{aligned}
B(x, y, z)= & \frac{1}{24}[f(x+y+z)+f(x-y-z) \\
& -f(x+y-z)-f(x-y+z)]
\end{aligned}
$$

for all $x, y, z \in E_{1}$, is symmetric for each fixed one variable and additive for each fixed two variables. It is easy to see that the functional equation (2) is equivalent to a cubic functional equation

$$
C(2 x+y)+C(x-y)+3 C(y)=3 C(x+y)+6 C(x)
$$


and every solution of the cubic functional equation is said to be a cubic mapping [11]. Najati [9] investigated the following generalized cubic functional equation:

$$
f(s x+y)+f(s x-y)=s f(x+y)+s f(x-y)+2\left(s^{3}-s\right) f(x)
$$

for a positive integers $s \geq 2$. Also, Jun and Kim [7] proved the Hyers-UlamRassias stability of a Euler-Lagrange type cubic mapping as follows:

$$
\begin{aligned}
& f(s x+y)+f(x+s y) \\
& =(s+1)(s-1)^{2}[f(x)+f(y)]+s(s+1) f(x+y),
\end{aligned}
$$

where $s \in \mathbb{Z}(s \neq 0, \pm 1)$.

In this paper, we deal with the following cubic functional equation with three variables which is equivalent to (2),

$$
\begin{aligned}
& 4\{f(2 x+y+z)+f(x+2 y+z)+f(x+y+2 z)\}= \\
& 27 f(x+y+z)+f(-x+y+z)+f(x-y+z) \\
& +f(x+y-z)+12\{f(x)+f(y)+f(z)\} .
\end{aligned}
$$

It is easy to see that the function $f(x)=c x^{3}$ is a solution of the above functional equation. We will investigate the stability of a cubic Lie $*$-derivations associated with the given functional equation on normed algebras.

\section{General Solution}

Let $\mathbb{R}^{+}$denote the set of all nonnegative real numbers and let both $E_{1}$ and $E_{2}$ be real vector spaces. We here present the general solution of (3).

Theorem 1. A function $f: E_{1} \rightarrow E_{2}$ satisfies the functional equation (2) if and only if $f: E_{1} \rightarrow E_{2}$ satisfies the functional (3). Therefore, every solution of functional equations (3) is also a cubic function.

Proof. Assume that $f: E_{1} \rightarrow E_{2}$ satisfy the functional equation (3). Putting $x=y=z=0$ in (2), we get $f(0)=0$. Setting $y=z=0$ in (3), we have

$$
4 f(2 x)=33 f(x)+f(-x) .
$$

for all $x \in E_{1}$. Letting $y=x, z=0$ in (3), we obtain

$$
f(3 x)=3 f(2 x)+3 f(x)
$$


for all $x \in E_{1}$. Replacing $y$ and $z$ by $x$ and $x$ in (3) respectively, we get

$$
12 f(4 x)=27 f(3 x)+39 f(x)
$$

for all $x \in E_{1}$. Using (5) and (6), we have

$$
12 f(4 x)=81 f(2 x)+120 f(x)
$$

for all $x \in E_{1}$. Associating (4) and (7), we obtain

$$
12 f(4 x)=\frac{3153}{4} f(x)+\frac{81}{4} f(-x)
$$

for all $x \in E_{1}$. Also,

$$
12 f(4 x)=3(33 f(2 x)+f(-2 x))=\frac{3270}{4} f(x)+\frac{198}{4} f(-x)
$$

for all $x \in E_{1}$, by using (4). So $f(-x)=-f(x)$ for all $x \in E_{1}$. Letting $z=0$ in (3), we have

$$
f(2 x+y)+f(x+2 y)=6 f(x+y)+3 f(x)+3 f(y)
$$

for all $x, y \in E_{1}$. Replacing $x$ by $x-y$, we get

$$
f(2 x-y)=-f(x+y)+3 f(x-y)+6 f(x)+3 f(y)
$$

for all $x, y \in E_{1}$. Taking $y$ into $-y$ in (11) and adding (11), we have (2).

Conversely, if $f: E_{1} \rightarrow E_{2}$ satisfies (2), then by [8], we can get a function $B: E_{1} \times E_{1} \times E_{1} \rightarrow E_{2}$ such that $f(x)=B(x, x, x)$ for all $x \in E_{1}$, and $B$ is symmetric for fixed one variable and $B$ is additive for fixed two variables. It is obvious that $f$ satisfies (3).

As the above result and Corollary 2.4 of [7], we obtain the following Corollary.

Corollary 2. If a mapping $f: \mathbb{R} \rightarrow \mathbb{R}$ is measurable which satisfies (3), then $f$ is continuous on $\mathbb{R}$ and $f(x)=f(1) x^{3}$ for all $x \in \mathbb{R}$. 


\section{Cubic Lie $*$-Derivations}

Throughout this section, we assume that $A$ is a complex normed $*$-algebra and $M$ is a Banach $A$-module. We will use the same symbol $\|\cdot\|$ as norms on a normed algebra $A$ and a normed $A$-bimodule $M$. A mapping $f: A \rightarrow M$ is a cubic homogeneous mapping if $f(\mu a)=\mu^{3} f(a)$, for all $a \in A$ and $\mu \in \mathbb{C}$. A cubic hoogeneous mapping $f: A \rightarrow M$ is called a cubic derivation if

$$
f(x y)=f(x) y^{3}+x^{3} f(y)
$$

holds for all $x, y \in A$. For all $x, y \in A$, the symbol $[x, y]$ will denote the commutator $x y-y x$. We say that a cubic homogeneous mapping $f: A \rightarrow M$ is a cubic Lie derivation if

$$
f([x, y])=\left[f(x), y^{3}\right]+\left[x^{3}, f(y)\right]
$$

for all $x, y \in A$. In addition, if $f$ satisfies in condition $f\left(x^{*}\right)=f(x)^{*}$ for all $x \in A$, then it is called the cubic Lie $*$-derivation. In the following, $\mathbb{T}^{1}$ will stand for the set of all complex units, that is,

$$
\mathbb{T}^{1}=\{\mu \in \mathbb{C}|| \mu \mid=1\} .
$$

For the given mapping $f: A \rightarrow M$, we consider

$$
\begin{aligned}
\triangle_{\mu} f(x, y, z):= & f(2 \mu x+\mu y+\mu z)+f(\mu x+2 \mu y+\mu z) \\
& +f(\mu x+\mu y+2 \mu z)-\frac{27}{4} \mu^{3} f(x+y+z) \\
& -\frac{1}{4} \mu^{3}\{f(-x+y+z)+f(x-y+z) \\
& +f(x+y-z)\}-3 \mu^{3}\{f(x)+f(y)+f(z)\}, \\
\triangle f(x, y):= & f([x, y])-\left[f(x), y^{3}\right]-\left[x^{3}, f(y)\right]
\end{aligned}
$$

for all $x, y, z \in A, \mu \in \mathbb{C}$.

Theorem 3. Let $\varphi: A^{3} \rightarrow \mathbb{R}^{+}$and $\psi: A^{3} \rightarrow \mathbb{R}^{+}$be functions such that

$$
\tilde{\varphi}(x):=\sum_{n=0}^{\infty} \frac{1}{8^{n}} \varphi\left(2^{n} x, 0,0\right)<\infty
$$

and

$$
\lim _{n \rightarrow \infty} \frac{1}{8^{n}} \varphi\left(2^{n} x, 2^{n} y, 2^{n} z\right)=0
$$




$$
\lim _{n \rightarrow \infty} \frac{1}{8^{n}} \psi\left(2^{n} x, 2^{n} y, 2^{n} z\right)<\infty
$$

for all $x, y, z \in A$. Suppose that $f: A \rightarrow M$ is a mapping with $f(0)=0$ such that

$$
\left\|\triangle_{\mu} f(x, y, z)\right\| \leq \varphi(x, y, z)
$$

and

$$
\left\|\triangle f(x, y)+f\left(z^{*}\right)-f(z)^{*}\right\| \leq \psi(x, y, z)
$$

for all $\mu \in \mathbb{T}_{\frac{1}{n_{0}}}^{1}=\left\{e^{i \theta} \mid 0 \leq \theta \leq \frac{2 \pi}{n_{0}}\right\}$ and all $x, y, z \in A$ in which $n_{0} \in \mathbb{N}$. Also, if $f$ is measurable or $f(t x)$ is continuous in $t \in \mathbb{R}$ for each fixed $x \in A$, then there exists a unique cubic Lie $*$-derivation $T: A \rightarrow M$ satisfying

$$
\|f(x)-T(x)\| \leq \frac{1}{8}\left(\frac{\tilde{\varphi}(x)+\tilde{\varphi}(-x)}{2}\right)+\frac{2}{17}\left(\frac{\tilde{\varphi}(x)+\tilde{\varphi}(-x)}{2}\right)
$$

for all $x \in A$, where $\tilde{\tilde{\varphi}}(x)=\sum_{n=0}^{\infty}\left(\frac{2}{17}\right)^{n} \varphi\left(2^{n} x, 0,0\right)$.

Proof. Let $y=z=0$ and $\mu=1$ in (15), we have

$$
\left\|f(2 x)-\frac{33}{4} f(x)-\frac{1}{4} f(-x)\right\| \leq \varphi(x, 0,0)
$$

for all $x \in A$. One can easily get

$$
\begin{aligned}
& \left\|\frac{1}{8} f_{o}(2 x)-f_{o}(x)\right\| \leq \frac{1}{8}\left(\frac{\varphi(x, 0,0)+\varphi(-x, 0,0)}{2}\right) \\
& \left\|\frac{2}{17} f_{e}(2 x)-f_{e}(x)\right\| \leq \frac{2}{17}\left(\frac{\varphi(x, 0,0)+\varphi(-x, 0,0)}{2}\right)
\end{aligned}
$$

where $f_{e}(x)=\frac{f(x)+f(-x)}{2}$ and $f_{o}(x)=\frac{f(x)-f(-x)}{2}$ for all $x \in A$. Using the induction, it is easy to show that

$$
\begin{aligned}
& \left\|\frac{1}{8^{t}} f_{o}\left(2^{t} x\right)-\frac{1}{8^{k}} f_{o}\left(2^{k} x\right)\right\| \\
& \quad \leq \frac{1}{8} \sum_{j=k}^{t-1} \frac{1}{8^{j}}\left(\frac{\varphi\left(2^{j} x, 0,0\right)+\varphi\left(-2^{j} x, 0,0\right)}{2}\right) \\
& \left\|\left(\frac{2}{17}\right)^{t} f_{e}\left(2^{t} x\right)-\left(\frac{2}{17}\right)^{k} f_{e}\left(2^{k} x\right)\right\|
\end{aligned}
$$




$$
\leq \frac{2}{17} \sum_{j=k}^{t-1}\left(\frac{2}{17}\right)^{j}\left(\frac{\varphi\left(2^{j} x, 0,0\right)+\varphi\left(-2^{j} x, 0,0\right)}{2}\right)
$$

for all $t>k>0$ and $x \in A$. The inequality (12) and (19), (20) imply that the sequences $\left\{\frac{1}{8^{n}} f_{e}\left(2^{n} x\right)\right\}$ and $\left\{\left(\frac{2}{17}\right)^{n} f_{e}\left(2^{n} x\right)\right\}$ are Cauchy. Since $M$ is complete, these sequences are convergent. Hence we can define two mappings $T_{o}, T_{e}$ as

$$
T_{o}(x)=\lim _{n \rightarrow \infty} \frac{1}{8^{n}} f_{o}\left(2^{n} x\right), \quad T_{e}(x)=\lim _{n \rightarrow \infty}\left(\frac{2}{17}\right)^{n} f_{e}\left(2^{n} x\right)
$$

for all $x \in X$. By taking $t \rightarrow \infty$ and $k=0$ in the inequalities (19) and (3.9), we have

$$
\begin{aligned}
& \left\|f_{o}(x)-T_{o}(x)\right\| \leq \frac{1}{8}\left(\frac{\tilde{\varphi}(x)+\tilde{\varphi}(-x)}{2}\right), \\
& \left\|f_{e}(x)-T_{e}(x)\right\| \leq \frac{2}{17}\left(\frac{\tilde{\tilde{\varphi}}(x)+\tilde{\varphi}(-x)}{2}\right)
\end{aligned}
$$

for all $x \in A$. If we define $T=T_{o}+T_{e}$, we get (17).

Now, we will show that the mapping $T$ is a unique cubic Lie $*$-derivation such that the inequality (17) holds for all $x \in A$. We note that

$$
\begin{aligned}
& \left\|\triangle_{\mu} T(x, y, z)\right\| \leq\left\|\triangle_{\mu} T_{o}(x, y, z)\right\|+\left\|\triangle_{\mu} T_{e}(x, y, z)\right\| \\
& \leq \lim _{n \rightarrow \infty} \frac{2}{8^{n}} \frac{\varphi\left(2^{n} x, 2^{n} y, 2^{n} z\right)+\varphi\left(-2^{n} x,-2^{n} y,-2^{n} z\right)}{2}=0
\end{aligned}
$$

for all $x, y, z \in A$ and $\mu \in \mathbb{T}_{\frac{1}{n_{0}}}^{1}$. By taking $\mu=1$ in the inequality (21), it follows that the mapping $T$ is a cubic mapping. Also, the inequality (21) implies that $\triangle_{\mu} T(x, y, z)=0$ for all $x, y, z \in A$. Hence

$$
T(\mu x)=\mu^{3} T(x)
$$

for all $x \in A$ and $\mu \in \mu_{1} \in \mathbb{T}_{\frac{1}{n_{0}}}^{1}$. Let $\mu \in \mathbb{T}^{1}=\{\lambda \in \mathbb{C}|| \lambda \mid=1\}$. Then $\mu=e^{i \theta}$, where $0 \leq \theta \leq 2 \pi$. Let $\mu_{1}=\mu^{\frac{1}{n_{0}}}=e^{\frac{i \theta}{n_{o}}}$. So we have $\mu_{1} \in \mathbb{T}_{\frac{1}{n_{0}}}^{1}$. Then

$$
L(\mu x)=L\left(\mu_{1}^{n_{0}} x\right)=\mu^{3 n_{0}} L(x)=\mu^{3} L(x)
$$

for all $\mu \in \mathbb{T}^{1}$ and $x \in A$. For any continuous linear functional $L$ defined on $A$, let $\rho: \mathbb{R} \rightarrow \mathbb{R}$ be given by

$$
\rho(t):=L[T(t x)]
$$


for $t \in \mathbb{R}$, where $x$ is fixed. Then $\rho$ is a cubic mapping and, moreover, is also measurable since it is the pointwise limit of the sequence

$$
\rho_{n}(t):=8^{-n} L\left[f\left(2^{n} t x\right)\right] .
$$

Hence it has the form $\rho(t)=t^{3} \rho(1)$ for all $t \in \mathbb{R}$ by Corollary 2.2. Therefore one obtains that for each fixed $x \in X$ and all $t \in \mathbb{R}$

$$
L[T(t x)]=\rho(t)=t^{3} \rho(1)=t^{3} L[T(x)]=L\left[t^{3} T(x)\right],
$$

which implies the condition

$$
T(t x)=t^{3} T(x), \quad \forall x \in A, \forall t \in \mathbb{R} .
$$

Let $\mu \in \mathbb{C}(\mu \neq 0)$. Then $\frac{\mu}{|\mu|} \in \mathbb{T}^{1}$. Hence

$$
T(\mu x)=T\left(\frac{\mu}{|\mu|}|\mu| x\right)=\left(\frac{\mu}{|\mu|}\right)^{3}|\mu|^{3} T(x)=\mu^{3} T(x)
$$

for all $x \in A$ and $\mu \in \mathbb{C}(\mu \neq 0)$. Since $x$ is an arbitrary element in $A$, we may conclude that $T$ is cubic homogeneous. Next, using (16), we have

$$
\frac{1}{8^{2 n}}\left\|\triangle f\left(2^{n} x, 2^{n} y\right)\right\| \leq \frac{\psi\left(2^{n} x, 2^{n} y, 0\right)}{8^{n}}
$$

for all $x, y \in A$. Taking the limit as $n$ tends to infinity, we have $\triangle T(x, y)=0$, for all $x, y \in A$. That is, $T$ is a cubic Lie derivation. Letting $x=y=0$ and replacing $z$ by $2^{n} x$ in the inequality (16), we get

$$
\left\|\frac{f\left(2^{n} z^{*}\right)}{8^{n}}-\frac{f\left(2^{n} z\right)^{*}}{8^{n}}\right\| \leq \frac{\psi\left(0,0,2^{n} z\right)}{8^{n}}
$$

for all $z \in A$. As $n \rightarrow \infty$ in the inequality (22), we have

$$
T\left(z^{*}\right)=T(z)^{*}
$$

for all $z \in A$. This means that $T$ is a cubic Lie $*$-derivation. Now, assume $T^{\prime}: A \rightarrow A$ is another cubic $*$-derivation satisfying the inequality (17). Then

$$
\begin{aligned}
\left\|T(x)-T^{\prime}(x)\right\| & =\frac{1}{8^{n}}\left\|T\left(2^{n} x\right)-T^{\prime}\left(2^{n} x\right)\right\| \\
& \leq \frac{1}{8^{n}}\left(\left\|T\left(2^{n} x\right)-f\left(2^{n} x\right)\right\|+\left\|f\left(2^{n} x\right)-T^{\prime}\left(2^{n} x\right)\right\|\right) \\
& \leq \frac{4}{8} \sum_{j=n}^{\infty} \frac{1}{8^{j}}\left(\frac{\varphi\left(2^{j} x, 0,0\right)+\varphi\left(-2^{j} x, 0,0\right)}{2}\right)
\end{aligned}
$$

which tends to zero as $n \rightarrow \infty$, for all $x \in A$. Thus $T(x)=T^{\prime}(x)$ for all $x \in A$. This proves the uniqueness of $T$. 
Corollary 4. Let $\varepsilon$ and $\varepsilon^{\prime}>0$. Suppose $f: A \rightarrow M$ is a mapping with $f(0)=0$ such that

$$
\left\|\triangle_{\mu} f(x, y, z)\right\| \leq \varepsilon
$$

and

$$
\left\|\triangle f(x, y)+f\left(z^{*}\right)-f(z)^{*}\right\| \leq \varepsilon^{\prime}
$$

for all $\mu \in \mathbb{T}_{\frac{1}{n_{0}}}^{1}=\left\{e^{i \theta} \mid 0 \leq \theta \leq \frac{2 \pi}{n_{0}}\right\}$ and all $x, y, z \in A$ in which $n_{0} \in \mathbb{N}$. Also, if $f$ is measurable or $f(t x)$ is continuous in $t \in \mathbb{R}$ for each fixed $x \in A$, then there exists a unique cubic Lie $*$-derivation $T: A \rightarrow M$ satisfying

$$
\|f(x)-T(x)\| \leq \frac{29}{105} \varepsilon
$$

for all $x \in A$.

Proof. Letting $\varphi(x, y, z)=\varepsilon, \psi(x, y)=\varepsilon^{\prime}$ and applying Theorem 3, we get the desired result, as claimed.

Corollary 5. Let $\theta, \theta^{\prime}>0$ and $0<r<3,0<r^{\prime}<3$. Suppose $f: A \rightarrow M$ is a mapping such that

$$
\left\|\triangle_{\mu} f(x, y, z)\right\| \leq \theta\left(\|x\|^{r}+\|y\|^{r}+\|z\|^{r}\right)
$$

and

$$
\left\|\triangle f(x, y)+f\left(z^{*}\right)-f(z)^{*}\right\| \leq \theta^{\prime}\left(\|x\|^{r^{\prime}}+\|y\|^{r^{\prime}}+\|z\|^{r^{\prime}}\right)
$$

for all $\mu \in \mathbb{T}_{\frac{1}{n_{0}}}^{1}=\left\{e^{i \theta} \mid 0 \leq \theta \leq \frac{2 \pi}{n_{0}}\right\}$ and all $a, b, c, x, y, z \in A$ in which $n_{0} \in \mathbb{N}$. Also, if $f$ is measurable or $f(t x)$ is continuous in $t \in \mathbb{R}$ for each fixed $x \in A$, then there exists a unique cubic Lie $*$-derivation $T: A \rightarrow M$ satisfying

$$
\|f(x)-T(x)\| \leq \frac{\theta\|x\|^{r}}{8-2^{r}}+\frac{\theta\|x\|^{r}}{\frac{17}{2}-2^{r}}
$$

for all $x \in A$.

Proof. Letting $\varphi(x, y, z)=\theta\left(\|x\|^{r}+\|y\|^{r}+\|z\|^{r}\right), \psi(x, y)=\theta^{\prime}\left(\|x\|^{r^{\prime}}+\|y\|^{r^{\prime}}+\right.$ $\|z\|^{r^{\prime}}$ ) and applying Theorem 3 , we get the desired result, as claimed. 
Theorem 6. Let $\phi: A^{3} \rightarrow \mathbb{R}^{+}$and $\psi^{\prime}: A^{3} \rightarrow \mathbb{R}^{+}$be functions such that

$$
\tilde{\tilde{\phi}}(x):=\sum_{n=1}^{\infty}\left(\frac{17}{2}\right)^{n} \phi\left(\frac{x}{2^{n}}, 0,0\right)<\infty
$$

and

$$
\begin{aligned}
& \lim _{n \rightarrow \infty}\left(\frac{17}{2}\right)^{n} \phi\left(\frac{x}{2^{n}}, \frac{y}{2^{n}}, \frac{z}{2^{n}}\right)=0, \\
& \lim _{n \rightarrow \infty} 8^{2 n} \psi^{\prime}\left(\frac{x}{2^{n}}, \frac{y}{2^{n}}, \frac{z}{2^{n}}\right)<\infty
\end{aligned}
$$

for all $x, y, z \in A$. Suppose that $f: A \rightarrow M$ is a mapping with $f(0)=0$ such that

$$
\left\|\triangle_{\mu} f(x, y, z)\right\| \leq \phi(x, y, z)
$$

and

$$
\left\|\triangle f(x, y)+f\left(z^{*}\right)-f(z)^{*}\right\| \leq \psi^{\prime}(x, y, z)
$$

for all $\mu \in \mathbb{T}_{\frac{1}{n_{0}}}^{1}=\left\{e^{i \theta} \mid 0 \leq \theta \leq \frac{2 \pi}{n_{0}}\right\}$ and all $x, y, z \in A$ in which $n_{0} \in \mathbb{N}$. Also, if $f$ is measurable or $f(t x)$ is continuous in $t \in \mathbb{R}$ for each fixed $x \in A$, then there exists a unique cubic Lie $*$-derivation $T: A \rightarrow M$ satisfying

$$
\|f(x)-T(x)\| \leq \frac{1}{8}\left(\frac{\tilde{\phi}(x)+\tilde{\phi}(-x)}{2}\right)+\frac{2}{17}\left(\frac{\tilde{\phi}(x)+\tilde{\tilde{\phi}}(-x)}{2}\right)
$$

for all $x \in A$, where $\tilde{\phi}(x)=\sum_{n=0}^{\infty} 8^{n} \phi\left(\frac{x}{2^{n}}, 0,0\right)$.

Proof. Using (18), we have

$$
\begin{gathered}
\left\|f_{o}(x)-8 f_{o}\left(\frac{x}{2}\right)\right\| \leq\left(\frac{\phi\left(\frac{x}{2^{j}}, 0,0\right)+\varphi\left(-\frac{x}{2}, 0,0\right)}{2}\right) \\
\left\|f_{e}(x)-\frac{17}{2} f_{e}\left(\frac{x}{2}\right)\right\| \leq\left(\frac{\phi\left(\frac{x}{2}, 0,0\right)+\phi\left(-\frac{x}{2}, 0,0\right)}{2}\right)
\end{gathered}
$$

for all $x \in A$. And using induction, we get

$$
\left\|8^{k} f_{o}\left(\frac{x}{2^{k}}\right)-8^{t} f_{o}\left(\frac{x}{2^{t}}\right)\right\| \leq \frac{1}{8} \sum_{j=k+1}^{t} 8^{j}\left(\frac{\phi\left(\frac{x}{2^{j}}, 0,0\right)+\phi\left(\frac{x}{2^{j}}, 0,0\right)}{2}\right)
$$




$$
\left\|\left(\frac{17}{2}\right)^{k} f_{e}\left(\frac{x}{2^{k}}\right)-\left(\frac{17}{2}\right)^{t} f_{e}\left(\frac{x}{2^{t}}\right)\right\| \leq \frac{2}{17} \sum_{j=k+1}^{t}\left(\frac{17}{2}\right)^{j}\left(\frac{\phi\left(\frac{x}{2^{j}}, 0,0\right)+\phi\left(\frac{x}{2^{j}}, 0,0\right)}{2}\right)
$$

for all $t>k>0$ and $x \in A$. Thus the sequences $\left\{8^{n} f_{o}\left(\frac{x}{2^{n}}\right)\right\}$ and $\left\{\left(\frac{17}{2}\right)^{n} f_{e}\left(\frac{x}{2^{n}}\right)\right\}$ are Cauchy. So, we can define

$$
T_{o}(x)=\lim _{n \rightarrow \infty} 8^{n} f_{o}\left(\frac{x}{2^{n}}\right), \quad T_{e}(x)=\lim _{n \rightarrow \infty}\left\{\frac{17}{2}\right\}^{n} f_{e}\left(\frac{x}{2^{n}}\right)
$$

for all $x \in X$. If we take $T=T_{e}+T_{o}$, then we have (23). And

$$
\begin{aligned}
\left\|\triangle_{\mu} T(x, y, z)\right\| & \leq\left\|\triangle_{\mu} T_{o}(x, y, z)\right\|+\left\|\triangle_{\mu} T_{e}(x, y, z)\right\| \\
& \leq \lim _{n \rightarrow \infty} 2 \cdot\left(\frac{17}{2}\right)^{n} \frac{\phi\left(\frac{x}{2^{n}}, \frac{y}{2^{n}}, \frac{z}{2^{n}}\right)+\phi\left(-\frac{x}{2^{n}},-\frac{y}{2^{n}},-\frac{z}{2^{n}}\right)}{2}=0
\end{aligned}
$$

for all $x, y, z \in A$ and all $\mu \in \mathbb{T}_{\frac{1}{n_{0}}}^{1}$. By the similar way of proof of Theorem 3, we get the mapping $T$ is a unique cubic homogeneous. And we also have

$$
\begin{aligned}
& 8^{2 n}\left\|\triangle f\left(\frac{x}{2^{n}}, \frac{y}{2^{n}}\right)\right\| \leq 8^{2 n} \psi^{\prime}\left(\frac{x}{2^{n}}, \frac{y}{2^{n}}, 0\right) \\
& \left\|8^{n} f\left(\frac{z^{*}}{2^{n}}\right)-8^{n} f\left(\frac{z}{2^{n}}\right)^{*}\right\| \leq 8^{n} \psi^{\prime}\left(0,0, \frac{z}{2^{n}}\right)
\end{aligned}
$$

for all $x, y, z \in A$ and all $n \in \mathbb{N}$. The rest proof is similar to that of Theorem 3.

Corollary 7. Let $\theta, \theta^{\prime}>0$ and $r>\log _{2} \frac{17}{2}, r^{\prime}>6$. Suppose $f: A \rightarrow M$ is a mapping such that

$$
\left\|\triangle_{\mu} f(x, y, z)\right\| \leq \theta\left(\|x\|^{r}+\|y\|^{r}+\|z\|^{r}\right)
$$

and

$$
\left\|\triangle f(x, y)+f\left(z^{*}\right)-f(z)^{*}\right\| \leq \theta^{\prime}\left(\|x\|^{r^{\prime}}+\|y\|^{r^{\prime}}+\|z\|^{r^{\prime}}\right)
$$

for all $\mu \in \mathbb{T}_{\frac{1}{n_{0}}}^{1}=\left\{e^{i \theta} \mid 0 \leq \theta \leq \frac{2 \pi}{n_{0}}\right\}$ and all $x, y, z \in A$ in which $n_{0} \in \mathbb{N}$. Also, if $f$ is measurable or $f(t x)$ is continuous in $t \in \mathbb{R}$ for each fixed $x \in A$, then there exists a unique cubic Lie $*$-derivation $T: A \rightarrow M$ satisfying

$$
\|f(x)-T(x)\| \leq \frac{\theta\|x\|^{r}}{2^{r}-8}+\frac{\theta\|x\|^{r}}{2^{r}-\frac{17}{2}}
$$

for all $x \in A$. 
Proof. Letting $\phi(x, y, z)=\theta\left(\|x\|^{r}+\|y\|^{r}+\|z\|^{r}\right), \psi^{\prime}(x, y)=\theta^{\prime}\left(\|x\|^{r^{\prime}}+\right.$ $\|y\|^{r^{\prime}}+\|z\|^{r^{\prime}}$ ) and applying Theorem 6, we get the desired result, as claimed.

\section{Acknowledgements}

The authors would like to thank the referees for giving useful suggestions and for the improvement of this manuscript. This research was supported by Basic Science Research Program through the National Research Foundation of Korea (NRF) funded by the Ministry of Education (no. 2013R1A1A2A10004419).

\section{References}

[1] T. Aoki, On the stability of the linear transformation in Banach spaces, J. Math. Soc. Japan 2 (1950), 222-224.

[2] D. G. Bourgin, Classes of transformations and bordering transformations, Bull. Amer. Math. Soc., 57 (1951), 223-237.

[3] A. Fošner and M. Fošner, Approximate cubic Lie derivations, Abstract and Applied Analysis Vol. 2013, Article ID 425784, 5page, (2013)

[4] P. Găvruta A generalization of the Hyers-Ulam-Rassias stability of approximately additive mappings, J. Math. Anal. Appl. 184 (1994), 431-436

[5] D. H. Hyers, On the stability of the linear functional equation, Proc. Nat. Acad. Sci. U.S.A. 27 (1941), 222-224.

[6] S. Jang and C. Park, Approximate *-derivations and approximate quadratic *-derivations on $C^{*}$-algebra, J. Inequal. bf 2011 Article ID 55 (2011),

[7] K.W. Jun, H.M. Kim, On the stability of Euler-Lagrange type cubic functional equations in quasi-Banach spaces, J. Math. Anal. Appl. 332 (2007), 1335-1350.

[8] K.W. Jun, H.M. Kim, The generalized Hyers-Ulam-Rassias stability of a cubic functional equation, J. Math. Anal. Appl. 274 (2002), 867-878.

[9] A. Najati, The generalized Hyers-Ulam-Rassias stability of a cubic functional equation, Turk. J. Math. 31 (2007), 395-408 
[10] C. Park and A. Bodaghi, On the stability of *-derivations on Banach *-algebras, Adv. Diff. Equat. 2012 2012:138(2012)

[11] J.M. Rassias, Solution of the Ulam stability problem for cubic mappings, Glasnik Matem. 36 (2001), 63-72.

[12] Th. M. Rassias, On the stability of the linear mapping in Banach spaces, Proc. Amer. Math. Soc. 72 (1978), 297-300

[13] S. M. Ulam, Problems in Modern Mathematics, Chapter 6 Wiley Interscience, New York, 1964.

[14] S.Y. Yang, A. Bodaghi, K.A.M. Atan, Approximate cubic *-derivations on Banach *-algebra, Abstract and Applied Analysis, vol. 2012, Article ID 684179, 12pages, (2012). 
